\title{
Improved Control of a Virtual Prosthesis Using a Pattern Recognition Algorithm and an Interactive Training Environment in a Transhumeral Amputee Demonstrating Local Reinnervation
}

Ramly EP', Powell $\mathbf{M}^{2}$, Gilliam E ${ }^{1}$, Womack $I^{1}$, Dunbar $\mathrm{K}^{1}$, Yee $\mathbf{R}^{1}$, Goldstein $\mathrm{S}^{2}$, Beaulieu $\mathrm{B}^{2}$, Schreiber M1 and Chi $A^{* 1,2}$

${ }^{1}$ Department of Surgery, Portland

2Applied Physics Laboratory, Baltimore

*Corresponding author: Albert Chi, Department of Surgery, Portland

\section{ARTICLE INFO}

Received: 幽 May 20, 2019

Published: 蔧 May 28, 2019

Citation: Ramly EP, Powell M, Gilliam E, Womack I, Dunbar K, Yee R, Goldstein S, Beaulieu B, Schreiber M,Chi A. Improved Control of a Virtual Prosthesis Using a Pattern Recognition Algorithm and an Interactive Training Environment in a Transhumeral Amputee Demonstrating Local Reinnervation. Biomed J Sci \& Tech Res 18(3)-2019. BJSTR. MS.ID.003143.
ABSTRACT

Introduction: Improving the function of prosthetic arms remains challenging, especially for amputations at or proximal to the elbow. Myoelectric control via skin surface Electromyogram (EMG) detection is possible, but intuitive control of modern prostheses has not been fully achieved due to limited sites for input monitoring. We hypothesized that spontaneous postoperative local reinnervation by major motor nerves is possible. Like Targeted Muscle Reinnervation (TMR), this would permit coordinated muscular contractions correlating with phantom limb movements.

Methods: A patient with traumatic right transhumeral amputation was referred for TMR. On presentation, she already demonstrated evidence of local reinnervation. She was thus provided with specialized training using an interactive virtual environment to detect phantom limb movement. EMG recordings from 8 pairs of electrodes around the upper arm stump were analyzed with a pattern recognition algorithm. The interactive environment gave visual feedback in real time as the subject operated a virtual model of a highly dexterous prosthesis. Control of the virtual prosthesis before and after training was evaluated by movement completion percentage, movement completion time, and maintenance of the position achieved. Her results were compared to those of a cohort of transradial amputees undergoing the same training program.

Results: The patient's pattern of reinnervation and phantom limb control allowed for eleven unique hand, wrist, and elbow movements. Completion of the training program resulted in overall increase in EMG decoding accuracy between provided cues and decoded arm positions, as well as increase in virtual prosthesis movement completion percentage, and decrease in movement completion time. Except for a more modest improvement in movement completion time, the patient's results were comparable to those achieved by transradial amputees.

Conclusion: For one transhumeral amputee, local reinnervation coupled with an engineered system of pattern recognition and a virtual training program resulted in improved capabilities at the functional level of transradial amputees.

\section{Introduction}

Contemporary advances in amputation care include the development of myoelectric prostheses. Prosthetic control offering multiple degrees of freedom is made possible via detection of skin surface Electromyographic (EMG) signals. However, this is limited by the scarcity of muscle groups available to act as sites for input monitoring. Thus, despite advances in the devices themselves, achieving intuitive control of modern prostheses remains challenging. We hypothesized that the spontaneous development 
of post-amputation local reinnervation by major motor nerves is possible and can contribute to improved prosthetic control. Like Targeted Muscle Reinnervation (TMR), this would permit coordinated muscular contractions correlating to phantom limb movements [1]. This study evaluated the functional performance of a transhumeral amputee demonstrating signs of local reinnervation, before versus after training using a pattern recognition algorithm with a virtual interactive environment. The patient's performance was compared to that of a cohort of transradial amputees.

\section{Methods}

A patient fourteen years status post traumatic right transhumeral amputation was referred for evaluation for TMR. The patient had not undergone any additional surgical procedures. On initial evaluation, she was found to be demonstrating evidence of post-amputation local reinnervation. She was enrolled in a training program using a virtual environment to detect phantom limb movement. The program consisted of ten hour-long sessions focusing on the classification of discrete movements of her phantom limb.

EMG recordings from 8 pairs of electrodes around the upper arm stump were analyzed with a pattern recognition algorithm extracting features of signal absolute value, waveform length, and variance $[2,3]$. The program allows for on-screen portrayal of a highly dexterous virtual upper extremity prosthesis that interactively gives visual feedback in real time as the subject attempts to operate it. An on-screen prompt then appears, guiding the patient to perform certain movements such as closing or opening the hand, supination, pronation, etc. Movement of the on-screen prosthesis is controlled through decoding EMG signals generated by the patient while attempting to perform the prompted task. A confusion matrix is used to characterize the patient's ability to perform the intended action using the virtual environment.

Control of the virtual prosthesis before and after training was evaluated by movement completion percentage, movement completion time, and maintenance of the position achieved. The patient's results were compared to a cohort of transradial amputees undergoing the same training program. This study was approved by the Johns Hopkins Hospital Institutional Review Board.

\section{Results}

The patient's pattern of local reinnervation and corresponding phantom limb control allowed for discrimination of eleven unique hand, wrist, and elbow movements. There was significant improvement in the patient's performance after completion of the training program. For most discrete movements, improvement was noted in EMG decoding accuracy, i.e. the correlation between provided cues and decoded arm position (Figure 1).

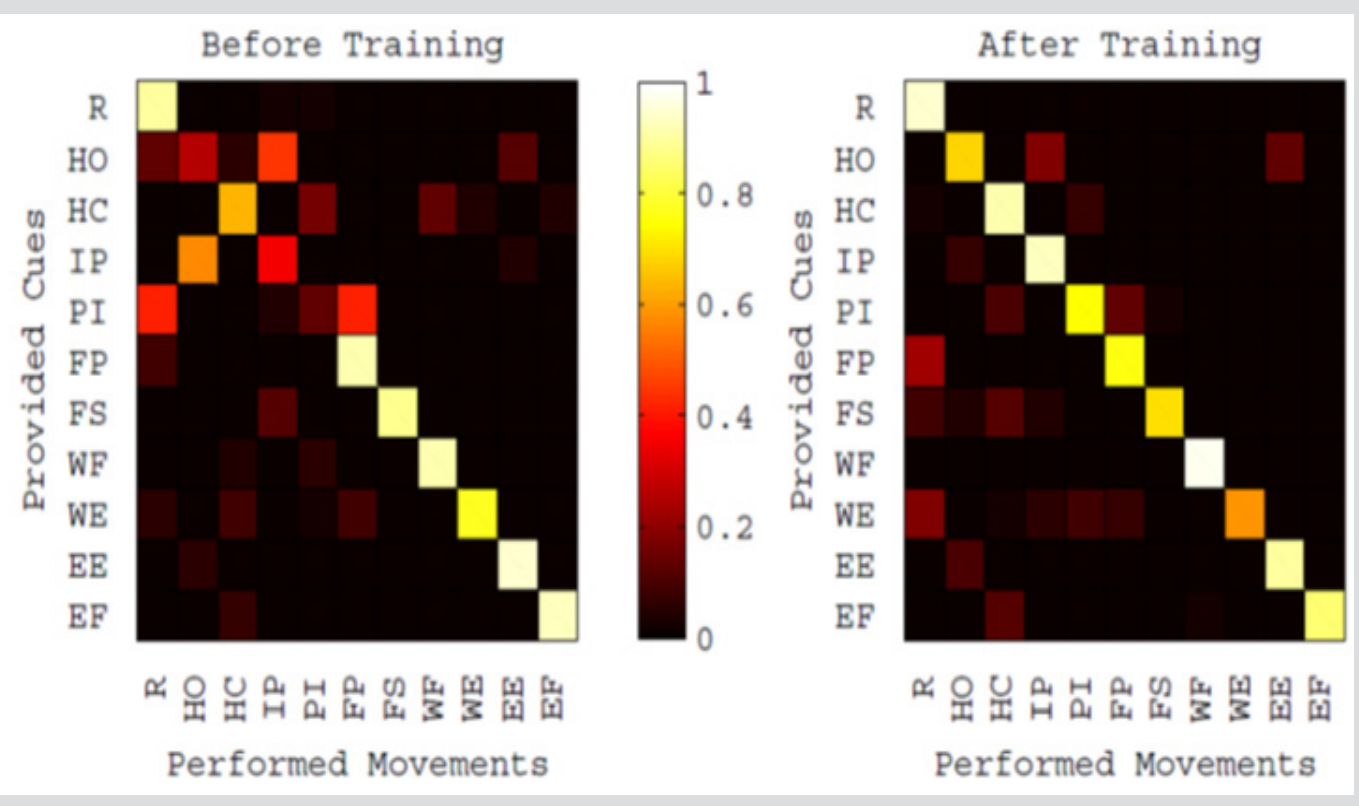

Figure 1: Example of confusion matrices visually representing EMG decoding accuracy, i.e. the correlation between provided EMG cues and decoded arm positions of the on-screen virtual prosthesis. The patient's pattern of local reinnervation and corresponding phantom limb control allowed for discrimination of eleven unique hand, wrist, and elbow movements. For the majority of discrete movements, improvement was noted in EMG decoding accuracy before versus after training.

Compared to the patient's own performance prior to enrolling in the program, completion of the training program resulted in overall increase in virtual prosthesis movement completion percentage, and decrease in movement completion time, as well as increase in post-selection classification accuracy (Figures 2A-2C). Except for a more modest improvement in movement completion time, the patient's results were comparable to those achieved by transradial amputees completing the same training program (Figures 2A-2C). 

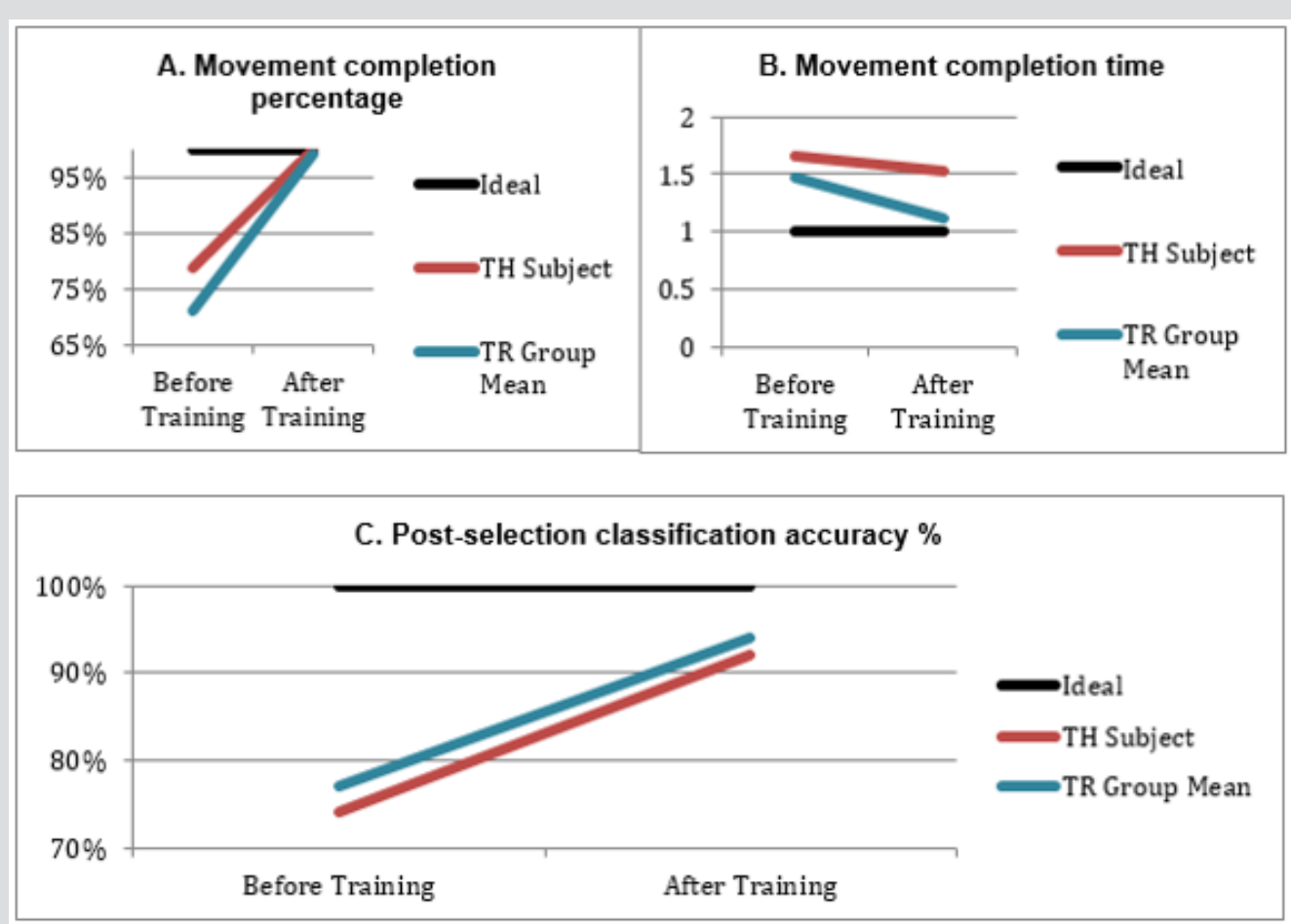

Figure 2: Performance of the Transhumeral Amputee (TH) compared to a cohort of Transradial Amputees (TR group mean), before and after training, using the same virtual interactive environment. A. Movement completion percentage. B. Movement completion time. C. Post-selection classification accuracy.

\section{Discussion}

TMR has been used both to treat post-amputation neuroma pain and to improve EMG signal transmission for myoelectric prosthetic control $[1,4]$. With this technique, nerves that once innervated muscles that have been amputated are re-routed towards alternative muscle units. The reinnervated units act as amplifiers of EMG signals, improving signal detection through surface EMG electrodes. The signals can then be measured and used to power myoelectric prosthetic devices [1]. More recently, high-density EMG arrays have been shown to improve signal decoding accuracy, such that a sense of the number of motor units activated and the relative power of the activation can be discerned [5]. Advances in TMR and pattern recognition systems promise to bring revolutionary improvements in myoelectric prosthetic control, including hand movements in patients with transhumeral amputation.

In our patient's case, we hypothesized that the spontaneous development of local reinnervation post-amputation allowed for the amplification of EMG signals in a TMR-like pattern. Local reinnervation coupled with an engineered system of pattern recognition and a virtual training program resulted in improved functional capabilities as demonstrated by an increase in virtual prosthesis movement completion percentage and decrease in movement completion time. Our findings suggest that a careful evaluation of EMG patterns in patients with upper extremity amputation can provide an opportunity to detect signs of spontaneous local reinnervation. Using a pattern recognition algorithm and proper training, local reinnervation configurations may be leveraged in appropriately selected patients to develop nuanced myoelectric prosthetic control without reverting to TMR. Further research is needed to elucidate the mechanisms of local reinnervation and technical considerations for promoting its development following primary amputation. We unfortunately do not have access to the operative record of the patient's posttraumatic surgical amputation performed 14 years prior to presentation. That would potentially have provided insight into how the nerves were handled during her initial procedure. Our small sample size also precludes the performance of more elaborate analyses. Future studies should focus on how to better leverage local reinnervation patterns for intuitive myoelectric prosthetic control.

\section{Conclusion}

For one transhumeral amputee demonstrating local reinnervation fourteen years status post amputation, EMG recordings coupled with an engineered system of pattern recognition and a virtual training program resulted in improved capabilities at the functional level of a cohort of transradial amputees. This suggests that intuitive control of myoelectric prostheses may be achieved by taking advantage of spontaneous local reinnervation patterns post-amputation, averting the need for additional surgery. 


\section{References}

1. Todd A Kuiken, Guanglin Li, Blair A Lock, Robert D Lipschutz, Laura A Miller, et al. (2009) Targeted muscle reinnervation for real-time myoelectric control of multifunction artificial arms. JAMA 301(6): 619628.

2. Powell MA, Kaliki RR, Thakor NV (2014) User training for pattern recognition-based myoelectric prostheses: improving phantom limb movement consistency and distinguishability. IEEE Trans Neural Syst Rehabil Eng 22(3): 522-532.

\section{ISSN: 2574-1241}

DOI: 10.26717/BJSTR.2019.18.003143

Albert Chi. Biomed J Sci \& Tech Res

This work is licensed under Creative Commons Attribution 4.0 License

Submission Link: https://biomedres.us/submit-manuscript.php
3. Powell MA, Thakor NV (2013) A training strategy for learning pattern recognition control for myoelectric prostheses. J Prosthet Orthot 25(1): $30-41$.

4. Souza JM, Cheesborough JE, Ko JH, Cho MS, Kuiken TA, et al. (2014) Targeted muscle reinnervation: a novel approach to postamputation neuroma pain. Clin Orthop Relat Res 472(10): 2984-2990.

5. Farina D, Rehbaum H, Holobar A, Vujaklija I, Jiang N, et al. (2014) Noninvasive, accurate assessment of the behavior of representative populations of motor units in targeted reinnervated muscles. IEEE Trans Neural Syst Rehabil Eng 22(4): 810-819.

$\begin{array}{ll}\text { BIOMEDICAL } & \text { Assets of Publishing with us } \\ \text { RESEARCHES } & \text { - Global archiving of articles } \\ & \text { - Immediate, unrestricted online access } \\ & \text { - Rigorous Peer Review Process } \\ \end{array}$

